\title{
Métodos de Gradiente Conjugado com Busca Linear Inexata ${ }^{1}$
}

B.P.B. SILVA, C.H. dos SANTOS, J.J. ROSSETTO, M. CARDIA, N.M.P. VOLPI, Y. JIAHONG, Departamento de Matemática, UFPR, 81531-990 Curitiba, PR, Brasil.

Este trabalho é dedicado à memória da Professora Beatriz P. de Barros e Silva falecida em 3 de abril de 2000.

Resumo. Neste trabalho apresentamos uma família de métodos de gradiente conjugado. Definimos uma direção de busca que depende de dois parâmetros e nem sempre satisfaz o critério das direções conjugadas, mas que, de certa forma, é uma aproximação da direção de Newton. Com uma escolha conveniente dos parâmetros, obtemos alguns dos métodos conhecidos na literatura. Testes computacionais foram realizados para comparar os novos métodos obtidos.

\section{Introdução}

Os métodos de gradiente conjugado têm despertado muito interesse para a resolução de problemas de minimização sem restrições de grande porte, devido a sua grande vantagem de não precisar guardar qualquer matriz, obtendo com isso um baixo custo computacional. Recentemente, vários autores têm considerado direções de busca que não satisfazem o critério das direções conjugadas, optando por aproximá-las, de alguma forma, da direção de Newton e em seguida fazendo uma busca linear inexata. Uma vasta literatura pode ser encontrada a respeito desse assunto, muitas das quais serão mencionadas no decorrer deste trabalho.

Seja o problema

$$
\underset{x \in \mathbb{R}^{n}}{\operatorname{minimizar}} f(x),
$$

onde $f: \mathbb{R}^{n} \longrightarrow \mathbb{R}$ é uma função com derivadas parciais contínuas.

Os métodos considerados neste trabalho geram os iterados

$$
\begin{gathered}
x_{k+1}=x_{k}+\alpha_{k} d_{k}, \\
d_{k+1}=-\theta_{k} g_{k+1}+\beta_{k} d_{k},
\end{gathered}
$$

\footnotetext{
${ }^{1}$ Apoio financeiro da FUNPAR
} 
onde $g_{k}$ denota $g\left(x_{k}\right)=\nabla f\left(x_{k}\right)$ e

$$
\beta_{k}=\frac{\left(\theta_{k} y_{k}-\frac{1}{s_{k}} p_{k}\right)^{t} g_{k+1}}{y_{k}^{t} d_{k}}
$$

com $y_{k}=g_{k+1}-g_{k}$ e $p_{k}=x_{k+1}-x_{k}=\alpha_{k} d_{k}$. Observemos que com uma escolha conveniente dos escalares $\theta_{k}$ e $s_{k}$ obtemos alguns dos métodos conhecidos na literatura. Seja $\theta_{k}=1$. Se $s_{k}=1$ então, substituindo (1.4) em (1.3), temos a direção apresentada por Perry em [8]. Se $s_{k} \longrightarrow \infty$, temos a direção de Hestenes-Stiefel [7]. Se a busca linear for exata, temos a direção de Polak-Ribière [9] e, se os sucessivos gradientes são ortogonais, temos a direção de Fletcher-Reeves [6]. Agora, se

$$
\theta_{k}=\frac{p_{k}^{t} p_{k}}{p_{k}^{t} y_{k}}
$$

e se $s_{k}=1$ então, (1.4) é a fórmula de Birgin-Martinez [2], que foi fortemente motivada pelos trabalhos de Barzilai-Borwein [1] e Raydan [10] e deu bons resultados computacionais.

Motivados pelo sucesso de Birgin-Martinez [2], considerando os bons resultados obtidos por Sherali-Ulular [13], que exigem que a direção de Perry [8] satisfaça equação

$$
s_{k} d_{k+1}=-H_{k+1}^{-1} g_{k+1},
$$

onde $H_{k+1}$ é a hessiana de $f$ em $x_{k+1}$, gerando $\beta_{k}=\left(y_{k}-\frac{1}{s_{k}} p_{k}\right)^{t} g_{k+1} / y_{k}^{t} d_{k}$ e inspirados no trabalho de Dai-Yuan-Yuan [3] que é uma generalização, sob o ponto de vista de interpolação, dos trabalhos de Barzilai-Borwein [1] e Raydan [10], tomaremos outros valores para $\theta_{k}$ e $s_{k}$. Seja $\theta_{k}$ dado por (1.5) e consideremos

$$
t_{k}=\theta_{k}^{-1}=\frac{p_{k}^{t} y_{k}}{p_{k}^{t} p_{k}} .
$$

O modelo quadrático

$$
q_{k+1}=f_{k+1}+\alpha g_{k+1}^{t} p_{k}+\frac{1}{2} t_{k} \alpha^{2}\left\|p_{k}\right\|^{2},
$$

onde $f_{k+1}=f\left(x_{k+1}\right)$, é uma aproximação para $f\left(x_{k+1}+\alpha p_{k}\right)$. É fácil ver que $t_{k}$ dado por (1.6) satisfaz a condição de interpolação

$$
\nabla q_{k+1}(-1)=g_{k}^{t} p_{k} .
$$

Portanto, $\theta_{k}$ dado por (1.5) é uma aproximação para a inversa da derivada direcional segunda de $f$ em $x_{k+1}$ ao longo de $p_{k}$.

Para qualquer $t_{k} \in \mathbb{R}$, o modelo quadrático (1.7) satisfaz as condições de interpolação

$$
q_{k+1}(0)=f_{k+1}
$$

e

$$
\nabla q_{k+1}(0)=g_{k+1}^{t} p_{k},
$$


Se (1.8) é substituída por uma outra condição de interpolação

$$
q_{k+1}(-1)=f_{k}
$$

então podemos obter de (1.7) que

$$
t_{k}=\frac{2\left(f_{k}-f_{k+1}+g_{k+1}^{t} p_{k}\right)}{\left\|p_{k}\right\|^{2}}
$$

donde

$$
\theta_{k}=\frac{p_{k}^{t} p_{k}}{2\left(f_{k}-f_{k+1}+g_{k+1}^{t} p_{k}\right)} .
$$

Além disso, podemos também construir um modelo cúbico

$$
c_{k+1}(\alpha)=f_{k+1}+\alpha g_{k+1}^{t} p_{k}+\frac{1}{2} t_{k} \alpha^{2}\left\|p_{k}\right\|^{2}+\xi_{k} \alpha^{3}
$$

tal que as condições (1.8)-(1.11) são todas satisfeitas, donde segue que

$$
t_{k}=\frac{6\left(f_{k}-f_{k+1}\right)+4 g_{k+1}^{t} p_{k}+2 g_{k}^{t} p_{k}}{p_{k}^{t} p_{k}}
$$

e, portanto,

$$
\theta_{k}=\frac{p_{k}^{t} p_{k}}{6\left(f_{K}-f_{k+1}\right)+4 g_{k+1}^{t} p_{k}+2 g_{k}^{t} p_{k}} .
$$

É fácil ver que as fórmulas (1.13) e (1.15) são idênticas a (1.5) se $f$ é quadrática no segmento de reta $x_{k}$ e $x_{k+1}$. Isto indica que se a função objetivo é quadrática (1.13) e (1.15) são exatamente os mesmos que (1.5). Então para uma função qualquer, como estamos usando as informações do valor da função e do valor do gradiente em $x_{k}$ e $x_{k+1}$ para derivar (1.13) e (1.15), é razoável esperar que estas duas fórmulas sejam melhores que (1.5).

Portanto, (1.3) e (1.4) nos dão uma estrutura unificada produzindo uma família de métodos de gradiente conjugado.

Observamos que as direções de Sherali-Ulular e de Birgin-Martinez serão paralelas se tomarmos o mesmo valor para os respectivos parâmetros $s_{k}$ e $\theta_{k}$.

\section{O Algoritmo}

Nesta seção descreveremos um método que é uma combinação das idéias dos algoritmos de gradiente conjugado escalado de Birgin-Martinez [2] e Sherali-Ulular $[13]$.

Algoritmo 2.1: $\operatorname{Dados} x_{o} \in \mathbb{R}^{n}, c=10^{-4}, r=0.9$ e $\epsilon>0$

\section{Inicialização \\ Faça}


Repita

$$
\begin{aligned}
& k=0 \\
& d_{0}=-g_{0}
\end{aligned}
$$

$$
\begin{aligned}
& \begin{array}{l}
\alpha_{k}=1 \text { ou } \\
\alpha=\alpha_{k}
\end{array}\left(\alpha_{k}=\left\{\begin{array}{ll}
1, & \text { se } k=0 \\
\alpha_{k-1} \frac{\left\|d_{k-1}\right\|}{\left\|d_{k}\right\|}, & \text { se } k \neq 0
\end{array}\right)\right. \\
& \text { Enquanto } f\left(x_{k}+\alpha d_{k}\right)>f\left(x_{k}\right)+c \alpha g_{k}^{t} d_{k} \text { ou } \\
& g\left(x_{k}+\alpha d_{k}\right)^{t} d_{k}<r g_{k}^{t} d_{k} \text { escolha } \sigma \in[0.1,0.5] \mathrm{e} \\
& \text { faça } \alpha=\sigma \alpha \text {. } \\
& \text { Fim } \\
& \alpha_{k}=\alpha \\
& x_{k+1}=x_{k}+\alpha_{k} d_{k} \\
& \beta_{k}=\frac{\left(\theta_{k} y_{k}-\frac{1}{s_{k}} p_{k}\right)^{t} g_{k+1}}{y_{k}^{t} d_{k}}, \text { com } \theta_{k}=1 \text { (ou dado por (1.5) ou (1.13) } \\
& \text { ou } \left.(1.15)) \text { e } s_{k}=1 \text { (ou } s_{k}=\alpha_{k}\right) \\
& d=-\theta_{k} g_{k+1}+\beta_{k} d_{k} \\
& \text { Se } d^{t} g_{k+1} \leq-10^{-3}\|d\|\left\|g_{k+1}\right\| \text { então } d_{k+1}=d \text {. } \\
& k=k+1 \\
& \text { Senão, } d_{k+1}=-\theta_{k} g_{k+1}
\end{aligned}
$$

Até que $\left\|g_{k}\right\|<\epsilon$

\section{Comentários:}

- A direção $d=-\theta_{k} g_{k+1}+\beta_{k} d_{k}$ não é necessariamente uma direção de descida. E esta é a razão que motivou as várias modificações da fórmula de Perry [8] feitas por Shanno [11].

- Estamos usando como reinicialização o mesmo critério ingênuo de BirginMartinez [2]. Com isso estamos garantindo que $d$ é uma direção de descida.

- Em Dennis-Schnabel [4] e Fletcher [5] é mostrado que um comprimento de passo $\alpha$ satisfazendo

$$
f\left(x_{k}+\alpha d_{k}\right) \leq f\left(x_{k}\right)+c \alpha g_{k}^{t} d_{k} \quad \text { e } \quad g\left(x_{k}+\alpha d_{k}\right)^{t} d_{k} \geq r g_{k}^{t} d_{k}
$$

sempre existe se $f$ é limitada inferiormente na direção $d_{k}$.

- Nas implementações também realizamos busca linear como em Shanno-Phua $[12]$.

\section{Resultados Numéricos e Conclusões}

Os resultados numéricos foram obtidos usando os problemas testes de Sherali-Ulular [13]. Consideramos oito métodos diferentes com dois critérios para escolha do tamanho de passo inicial na busca linear. Os métodos foram identificados como na Tabela 1 abaixo: 
Tabela 1

\begin{tabular}{||c|c|r|}
\hline MÉTODO & $\theta_{k}$ & $s_{k}$ \\
\hline$M_{1}$ & $p_{k}^{t} p_{k} / p_{k}^{t} y_{k}$ & $\alpha_{k}$ \\
\hline$M_{2}$ & $p_{k}^{t} p_{k} / 2\left(f_{k}-f_{k+1}+g_{k+1}^{t} p_{k}\right)$ & $\alpha_{k}$ \\
\hline$M_{3}$ & 1 & $\alpha_{k}$ \\
\hline$M_{4}$ & $p_{k}^{t} p_{k} /\left[6\left(f_{K}-f_{k+1}\right)+4 g_{k+1}^{t} p_{k}+2 g_{k}^{t} p_{k}\right]$ & $\alpha_{k}$ \\
\hline$M_{5}$ & $p_{k}^{t} p_{k} / p_{k}^{t} y_{k}$ & 1 \\
\hline$M_{6}$ & $p_{k}^{t} p_{k} / 2\left(f_{k}-f_{k+1}+g_{k+1}^{t} p_{k}\right)$ & 1 \\
\hline$M_{7}$ & 1 & 1 \\
\hline$M_{8}$ & $p_{k}^{t} p_{k} /\left[6\left(f_{K}-f_{k+1}\right)+4 g_{k+1}^{t} p_{k}+2 g_{k}^{t} p_{k}\right]$ & 1 \\
\hline
\end{tabular}

Os resultados da Tabela 2 correspondem a escolha do passo inicial na busca linear de $\alpha_{k}=1$ e os da Tabela 3 para $\alpha_{k}= \begin{cases}1 & \text { se } k=0, \\ \alpha_{k-1} \frac{\left\|d_{k-1}\right\|}{\left\|d_{k}\right\|} & \text { se } k \neq 0\end{cases}$

Os valores representados nas colunas das Tabelas 2 e 3 são o número de iterações e o número de chamadas da função, exceto na primeira coluna, onde $\mathrm{P}$ é o número do problema e n é o número de variáveis.

Tabela 2

\begin{tabular}{|c|c|c|c|c|c|c|c|c|}
\hline $\mathrm{P} / \mathrm{n}$ & $\mathrm{M} 1$ & $\mathrm{M} 2$ & $\mathrm{M} 3$ & $\mathrm{M} 4$ & $\mathrm{M} 5$ & $\mathrm{M} 6$ & $\mathrm{M} 7$ & $\mathrm{M} 8$ \\
\hline $1 / 2$ & $4 / 30$ & $4 / 30$ & $9 / 42$ & $5 / 36$ & $6 / 40$ & $6 / 40$ & $14 / 71$ & $16 / 79$ \\
\hline $2 / 2$ & $28 / 184$ & $27 / 202$ & $35 / 113$ & $24 / 116$ & $43 / 237$ & $37 / 208$ & $33 / 180$ & $14 / 102$ \\
\hline $3 / 4$ & $130 / 461$ & $102 / 366$ & $216 / 647$ & $118 / 357$ & $164 / 643$ & $254 / 857$ & $53 / 242$ & $250 / 836$ \\
\hline $4 / 4$ & $100 / 388$ & $169 / 630$ & $115 / 355$ & $116 / 436$ & $105 / 485$ & $78 / 307$ & $52 / 209$ & $108 / 420$ \\
\hline $5 / 2$ & $30 / 93$ & $11 / 44$ & $16 / 66$ & $9 / 55$ & $35 / 102$ & $9 / 39$ & $12 / 48$ & $12 / 71$ \\
\hline $6 / 5$ & $126 / 469$ & $137 / 470$ & $172 / 454$ & $203 / 597$ & $143 / 556$ & $131 / 500$ & $153 / 466$ & $97 / 374$ \\
\hline $6 / 10$ & $356 / 929$ & $343 / 885$ & $277 / 647$ & $271 / 759$ & $225 / 702$ & $252 / 779$ & $347 / 881$ & $227 / 713$ \\
\hline $7 / 20$ & $22 / 61$ & $25 / 64$ & $18 / 61$ & $25 / 56$ & $24 / 56$ & $17 / 51$ & $35 / 101$ & $34 / 87$ \\
\hline $7 / 40$ & $34 / 93$ & $36 / 92$ & $28 / 81$ & $38 / 101$ & $27 / 66$ & $36 / 93$ & $64 / 149$ & $40 / 108$ \\
\hline $7 / 200$ & $87 / 209$ & $96 / 255$ & $77 / 170$ & $110 / 261$ & $93 / 246$ & $79 / 220$ & $96 / 221$ & $90 / 235$ \\
\hline $8 / 3$ & $51 / 267$ & $94 / 333$ & $84 / 243$ & $56 / 218$ & $57 / 231$ & $83 / 279$ & $80 / 260$ & $70 / 275$ \\
\hline $9 / 5$ & $304 / 937$ & $266 / 874$ & $201 / 535$ & $209 / 657$ & $235 / 684$ & $271 / 886$ & $368 / 1024$ & $248 / 690$ \\
\hline $10 / 10$ & $8 / 60$ & $8 / 60$ & $7 / 54$ & $9 / 62$ & $9 / 65$ & $8 / 61$ & $9 / 87$ & $5 / 50$ \\
\hline
\end{tabular}

Tabela 3

\begin{tabular}{|c|c|c|c|c|c|c|c|c|}
\hline $\mathrm{P} / \mathrm{n}$ & $\mathrm{M} 1$ & $\mathrm{M} 2$ & $\mathrm{M} 3$ & $\mathrm{M} 4$ & $\mathrm{M} 5$ & $\mathrm{M} 6$ & $\mathrm{M} 7$ & $\mathrm{M} 8$ \\
\hline $1 / 2$ & $7 / 37$ & $7 / 37$ & $4 / 21$ & $11 / 54$ & $6 / 16$ & $6 / 16$ & $7 / 107$ & $7 / 20$ \\
\hline $2 / 2$ & $23 / 215$ & $28 / 190$ & $26 / 185$ & $22 / 153$ & $34 / 209$ & $28 / 137$ & $28 / 196$ & $21 / 124$ \\
\hline $3 / 4$ & $150 / 608$ & $434 / 1801$ & $160 / 1181$ & $115 / 450$ & $111 / 420$ & $54 / 198$ & $88 / 593$ & $234 / 868$ \\
\hline $4 / 4$ & $69 / 368$ & $97 / 547$ & $42 / 224$ & $82 / 377$ & $179 / 1043$ & $35 / 165$ & $105 / 511$ & $60 / 251$ \\
\hline $5 / 2$ & $17 / 59$ & $19 / 57$ & $14 / 41$ & $16 / 46$ & $22 / 70$ & $15 / 40$ & $15 / 43$ & $20 / 51$ \\
\hline $6 / 5$ & $299 / 1343$ & $355 / 1513$ & $222 / 1664$ & $112 / 390$ & $197 / 711$ & $173 / 632$ & $67 / 477$ & $121 / 390$ \\
\hline $6 / 10$ & $437 / 1845$ & $437 / 1916$ & $454 / 3487$ & $440 / 2938$ & $224 / 804$ & $255 / 868$ & $131 / 958$ & $193 / 791$ \\
\hline $7 / 20$ & $24 / 52$ & $27 / 61$ & $158 / 204$ & $25 / 57$ & $21 / 32$ & $24 / 35$ & $156 / 222$ & $26 / 62$ \\
\hline $7 / 40$ & $50 / 124$ & $35 / 85$ & $184 / 251$ & $38 / 104$ & $47 / 94$ & $35 / 58$ & $169 / 270$ & $29 / 69$ \\
\hline $7 / 200$ & $72 / 240$ & $71 / 241$ & $130 / 352$ & $70 / 206$ & $71 / 167$ & $66 / 163$ & $120 / 360$ & $64 / 160$ \\
\hline $8 / 3$ & $38 / 145$ & $68 / 231$ & $44 / 296$ & $65 / 215$ & $57 / 190$ & $59 / 212$ & $64 / 418$ & $65 / 185$ \\
\hline $9 / 5$ & $172 / 640$ & $223 / 859$ & $158 / 785$ & $175 / 296$ & $176 / 711$ & $156 / 636$ & $144 / 733$ & $157 / 568$ \\
\hline $10 / 10$ & $7 / 25$ & $7 / 25$ & $6 / 84$ & $7 / 25$ & $7 / 15$ & $7 / 15$ & $7 / 76$ & $7 / 15$ \\
\hline
\end{tabular}

Dos resultados numéricos obtidos concluímos que os métodos são competitivos, sendo que seu desempenho varia de acordo com o problema testado, com a escolha de um determinado parâmetro usado na busca linear (nossas tabelas apresentam os 
resultados apenas com valor $1 / 3$ para tal parâmetro) e com a escolha inicial para o tamanho do passo $\alpha_{k}$ na busca linear.

Os autores deste trabalho agradecem ao Prof. Dr. Jin Yun Yuan (UFPR) pelos comentários e sugestões recebidas.

\section{Referências}

[1] J. Barzilai, J.M. Borwein, Two-point stepsize gradient methods, IMA J. Num. Anal., 8 (1988), 141-148.

[2] E.G. Birgin, J.M. Martinez, A spectral conjugate gradiente method for unconstrained optimization, Technical Report, IMECC, Universidade de Campinas, 1998.

[3] Y.H. Dai, Y.J. Yuan, Y. Yuan, Modified two-point stepsize gradient methods for unconstrained optimization, Relatório Técnico, Departamento de Matemática, Universidade Federal do Paraná, 1998.

[4] J.E. Dennis Jr, R.B. Schnabel, "Numerical methods for unconstrained optimization and nonlinear equations", Prentice-Hall, Englewood Cliffs, 1983.

[5] R. Fletcher, "Practical methods of optimization", John-Wiley and Sons, New York, 1987.

[6] R. Fletcher, C.M. Reeves, Function minimization by conjugate gradient, Comput. J., 7 (1964), 149-154.

[7] M.R. Hestenes, E. Stiefel, Methods of conjugate gradients for solving linear systems, J. Res. Bur. Standards Sec., 48 (1952), 429-436.

[8] A. Perry, A modified conjugate gradient algorithm, Operations Research, 26 (1978), 1073-1078.

[9] E. Polak, G. Ribière, Note sur la convergence de méthodes de directions conjuguées, Revue Française d'Informatique et de Recherche Opérationnelle, 16 (1969), 35-43.

[10] M. Raydan, The Barzilai and Borwein gradient method for the large scale unconstrained minimization problem, SIAM J. Optim., 7 (1997), 26-33.

[11] D.F. Shanno, Conjugate gradiente method with inexact searches, Mathematics of Operations Research, 3 (1978), 244-256.

[12] D.F. Shanno, K.H. Phua, Minimization of unconstrained multivariate functions, ACM Transactions on Mathematical Software, 2 (1976), 87-94.

[13] H.D. Sherali, O. Ulular, Conjugate gradient methods using quasi-Newton updates with inexact line searches, Journal of Mathematical Analysis and Applications, 150 (1990), 359-377. 